Cahiers de philosophie de l'université de

CAHIERS DE PHILOSOPHIE
DE L L UNIVERSTIE DE CAEN

Caen

$50 \mid 2013$

Figures du cogito

\title{
René pense, donc Cartesius existe
}

Jaakko Hintikka

Traducteur : Xavier Kieft

CpenEdition

Journals

Édition électronique

URL : https://journals.openedition.org/cpuc/713

DOI : $10.4000 /$ cpuc. 713

ISSN : 2677-6529

Éditeur

Presses universitaires de Caen

Édition imprimée

Date de publication : 31 décembre 2013

Pagination : 107-120

ISBN : 978-2-84133-487-2

ISSN : 1282-6545

\section{Référence électronique}

Jaakko Hintikka, «René pense, donc Cartesius existe », Cahiers de philosophie de l'université de Caen

[En ligne], 50 | 2013, mis en ligne le 13 juin 2018, consulté le 02 février 2023. URL : http://

journals.openedition.org/cpuc/713 ; DOI : https://doi.org/10.4000/cpuc.713

\section{(c) (1) \&}

Creative Commons - Attribution - Pas d'Utilisation Commerciale 4.0 International - CC BY-NC 4.0

https://creativecommons.org/licenses/by-nc/4.0/ 


\section{René pense, donc Cartesius existe ${ }^{1}$}

\section{Cogito, ergo quis est?}

Celon une histoire célèbre, Mark Twain a fait taire les rumeurs $\mathcal{O}$ relatives à son décès en annonçant qu'elles étaient fortement exagérées. D’après une interprétation naturelle, Descartes se serait suggéré à lui-même en guise d'expérience de pensée qu'il n'existerait pas et, ce faisant, se serait prouvé à lui-même cette existence. Il y a des analogies et des dissemblances entre ces deux performances. J'ai fait usage de certaines analogies dans mon premier article sur le cogito cartésien il y a un demi-siècle ${ }^{2}$. Je maintiens toujours l'essentiel de ce que j'ai dit alors, même si j'ai réalisé que nommer l'argument de Descartes une "performance" avait en soi une assez faible valeur explicative. Ce terme souligne le fait que dans les deux exemples quelqu'un doit faire quelque chose lui-même pour établir la conviction qu'il a en vue. Mark Twain a lui-même dû procéder à une annonce. De même, bien que Descartes n'ait aucune relation avec la réalité extérieure, il doit non seulement examiner son inexistence possible, mais effectivement essayer de se l'affirmer à lui-même. Il ne lui suffit pas de contempler son existence dans un état d'esprit agnostique.

Une grande part de la problématique du cogito m’avait pourtant échappée. J'ai depuis réalisé qu'il s'y trouve plusieurs problèmes. D'une part, nous

1. En pensant à cet article et en l'écrivant, j'ai été encouragé et aidé par les commentaires d'Aaron Garrett et Bret Doyle. Je veux également adresser un remerciement à un inconnu. Je me souviens - malheureusement seulement vaguement - qu'il y a longtemps, un article a été soumis en vue d'une publication dans Synthese dont j'étais alors le directeur de publication. La thèse de cet article consistait dans une analogie entre les arguments de Descartes et de Gödel. Je n'avais alors pas été convaincu, et j'ai refusé l'article, dont je ne me rappelle plus l'auteur. J'espère qu'il ou elle prendra la parole et revendiquera pour son propre compte l'attribution de cette intuition intéressante.

2. J. Hintikka, «Cogito ergo sum: Inference or Performance?», Philosophical Review, vol. 71, $\mathrm{n}^{\circ}$ 1, 1962, p. 3-32; trad. fr. par P. Le Quellec-Wolff, Philosophie, no 6, 1985, p. 21-51. 
devons nous demander quel est le genre de l'entité dont Descartes espère prouver l'existence. Le chevalier inexistant d'Italo Calvino peut affirmer autant de fois qu'il le veut: «Je n'existe pas» en toute impunité ontologique, aussi longtemps qu'il ne renonce qu'à son existence en tant qu'objet physique ${ }^{3}$. Il ne constitue pas un contre-exemple de l'interprétation performative du cogito. Au contraire, son acte de langage même l'introduit dans le monde fictif de Calvino en tant que l'un de ses hôtes existant, en fait comme le personnage principal de l'ensemble de l'histoire. Ceci nous conduit à nous demander, comme je l'ai fait dans mon deuxième article sur le cogito, "Cogito, ergo quis est?», «Je pense, donc qui existe? » ${ }^{4}$. Et certes, s'il n’y a aucune réponse qui puisse être juste et que la seule conclusion que Cartesius aurait eu droit de tirer du cogito est «es denkt», alors la pensée seule se déploie. Descartes pourrait presque passer d'un seul coup de cogito ergo sum à sum res cogitans.

Cette question du «qui» est également pertinente parce que toutes les différences entre les différents objets ne comptent pas. Par exemple, je ne m'intéresse pas aux contrastes entre le mode d'existence des objets physiques et une sorte d'existence éthérée mais personnelle qui a été illustrée par l'homme invisible de H. G. Wells. Ce qui compte est ce qui est pris en charge par les questions du type «qui? », «quoi? », «quand?» et «où? : le mode d'identification de l'existant présumé. Quine avait raison: il n'y a pas d'entité sans identité. Nous avons besoin d'un système de principes d'identification.

\section{Les identités comme rôles}

L'importance de tels systèmes d'identification dans notre sémantique actuelle et son indépendance vis-à-vis du système de référence ne sont toujours pas estimées à leur juste valeur par les philosophes, malgré le fait parfaitement évident que dans notre pratique sémantique actuelle nous faisons tacitement confiance à plus d'un système d'identification fonctionnant en tandem avec le même système de référence (descriptive).

La possibilité d'une identification duale est illustrée par une analogie que Descartes aurait peut-être appréciée. Dans une pièce amateur, les dramatis personce ont une entité double. D'un côté, ils sont les personnages du drame qui se déroule sur la scène, peut-être des rois, des reines, des chevaliers et ainsi de suite. Mais d'un autre côté, quand ils passent la porte du théâtre, ils

3. Voir I. Calvino, Il cavaliere inesistente, Turin, Einaudi (Coralli ; 108), 1959; trad. angl. The Nonexistent Knight and the Cloven Viscount, Londres, Collins, 1962; trad. fr. Le chevalier inexistant, M. Javion (trad.), Paris, Gallimard (Folio; 5459), 2012.

4. J. Hintikka, «Cogito, ergo quis est?», Revue internationale de philosophie, t. 50, nº 195, 1996, p. 5-21. 
n'ont que leur identité de citoyen ordinaire, peut-être comme les ouvriers ou paysans proverbiaux 5 .

Ces acteurs peuvent sans troubler personne utiliser le même langage sur scène et en dehors de la pièce de théâtre. Mais l'auditoire (ou n'importe qui d'autre) ne peut pas tirer de conclusions relatives à leur vie de tous les jours à partir de ce que les acteurs disent et font dans le cadre de la pièce et vice versa. Ce qui est particulièrement important ici, c'est que ce double discours s'étend aux quantificateurs et est d'une certaine manière codifié en eux. Un logicien modal reconnaîtra immédiatement quels sont les quantificateurs qui se rapportent à un mode d'identification. Dire que «tous les rois sont des hommes» dans une pièce de théâtre fait référence à un ordre d'objets différent en dehors de la pièce et dans le cadre de celle-ci. Ceci comprend notamment le quantificateur existentiel qui, dans notre logique symbolique commune, détient un monopole virtuel quand il s'agit d'exprimer l'existence.

La raison de cette relation spéciale des quantificateurs à un mode d'identification est évidente dans une perspective de mondes possibles. Les valeurs des variables quantifiées sont des individus (ou des entités d'un autre type logique) bien définis. Cette bonne détermination implique qu'ils supportent la même identité dans différents «mondes possibles » (scénarios possibles). C'est pourquoi on doit, pour interpréter un quantificateur, avoir un critère d'identité à travers différents mondes (scénarios), en d'autres termes, un mode particulier d'identification. Ce mode est ce qui est fourni par un système d'identification. Ce n'est pas fourni en ne spécifiant que la référence ou en spécifiant une à une les expressions d'une entité dans les différents mondes possibles.

Un mode d'identification peut-être conçu comme un canevas ou une «carte» (Peirce a utilisé le mot « diagramme») qui est partagé par les scénarios (mondes) pertinents. Il est clair qu'une telle «carte» peut être dessinée de différentes manières.

\section{Identification perspective $v s$ identification publique}

En épistémologie et en métaphysique, nous ne traitons pas d'univers fictionnels dans le sens qu'a ce mot dans une pièce de théâtre. Mais dans un sens «le monde entier est une scène» pour un épistémologue. Un épistémologue ou un sémanticien peut prélever un épisode du monde réel (ou

5. J. Hintikka emploie l'expression «Tinkers, tailors, soldiers, sailors» par laquelle on désigne au début d'une comptine les bricoleurs, les tailleurs, les soldats et les marins. L'expression est idiomatique sans avoir d'équivalent strict en français (NDT). 
d'un monde imaginaire) et décider d'en identifier les agents par les termes qui désignent leurs rôles dans cet épisode au lieu d'utiliser leurs noms, comme un commentateur sportif qui décrit ce que font «le lanceur» ou «l'arrêt-court» au base-ball. Même si les mêmes joueurs ou autres agents peuvent être identifiés par leur nom, on peut parler des événements de la vie réelle comme s'ils étaient des pièces de théâtre ou des épisodes de série télévisée, ou même d'interminables soap-opéras. Le plus simple canevas d'identification de ce type est l'espace visuel que quelqu'un embrasse à un moment donné. Vous pouvez identifier des objets, des lieux, des événements, etc. par le rôle qu'ils assument dans le drame (ou la comédie) du passé qu'on se remémore personnellement.

De tels modes d'identification, centrés sur le sujet, sont nommés perspectifs. Ils doivent être distingués des identifications qui sont nommées publiques ou modes centrés sur l'objet. La différence est aisément explicitée quand on pense au monde en faisant référence aux anciennes pièces de théâtre dans lesquelles les acteurs portaient un masque. L'identification publique concerne un acteur après qu'il a ôté son masque, déposé sa persona perspective.

Ces concepts et distinctions ont été expliqués en détail dans mes premiers travaux. Ils se rapportent à des distinctions familières aux neuroscientifiques. Par exemple, dans la cognition visuelle, le contraste entre l'identification perspective et l'identification publique est manifesté par un contraste entre le (mal nommé) système «où? » et le système «quoi?». Dans le premier, l'espace visuel de quelqu'un est le canevas de l'identification. Dans le second, le canevas (si ce mot est le plus convenable ici) peut être conçu comme celui dans lequel les recenseurs essaient de placer les habitants d'une région. Psychologiquement parlant, il s'agit du canevas tacite que nous utilisons dans la reconnaissance d'un objet visuel.

La distinction entre l'identification publique et l'identification perspective se manifeste notamment dans l'ambiguïté opérationnelle des questions «qui... ?». Imaginez que j'assiste à une assemblée de grands financiers et que je demande: «qui est ce monsieur?». Je recevrai peut-être la réponse: «Ce monsieur est Georges Soros». Un participant plus averti saura peut-être tout ce qu'il faut du fameux investisseur mais ne saura pourtant pas le reconnaître et demandera peut-être: «Où se trouve Georges Soros?» Une réponse pourra être formulée par les mêmes mots: «Ce monsieur est Georges Soros».

Évidemment, la force de celle-ci et la forme énonciatrice diffèrent dans les deux circonstances. Un logicien épistémique ne rencontrera aucune difficulté à situer la différence dans l'ambiguïté des questions «qui... ?» et même à la situer dans une différence entre deux paires de quantificateurs différentes, relatives à deux modes d'identification différents. 
Il est tentant ici de comparer la distinction entre les objets identifiés de façon perspective et les objets publiquement identifiés avec la distinction entre les objets phénoménologiques et physiques (naturels). Je crois qu'il y a bien ici une connexion profonde. En effet, en phénoménologie comme dans la théorie des objets d'accointance de Russell, nous traitons en dernière analyse de deux sortes d'individuation plutôt que de deux espèces d'objets. Malheureusement, la relation entre les deux contrastes est trop pleine de subtilités pour assumer ici une fonction explicative sans explication supplémentaire. J'ai traité quelques-unes d'entre elles dans les essais rassemblés sous le titre Paradigms for Language Theory and Other Essays ${ }^{6}$.

Cette différence montre de manière dramatique que nous devons toujours nous fier à une sémantique actuelle pour situer quelqu'un dans un système d'identification, et pas seulement à un système de référence. Car les références de nos expressions linguistiques sont évidemment les mêmes quel que soit le mode d'identification que nous employons. Il s'avère même que dans le cas de la cognition visuelle les deux systèmes d'identification sont traités par deux centres du cerveau anatomiquement distincts. En termes de linguistique, toute question «qui? » ou «quoi? ? présuppose un mode d'identification particulier. Autrement, il demeure ambigu de la même façon que dans mon échantillon d'enquête sur «Qui est Georges Soros?».

\section{Le «Je» de Descartes n'est-il que perspectif?}

Ce que tout cela implique pour le cogito est clair. En considérant sa propre existence, Descartes doit tacitement assumer un type d'indentification particulier. Autrement les critiques de Lichtenberg s'appliqueraient sans aucune pitié sémantique.

Il est également clair que l'objet dont Descartes peut espérer prouver l'existence en premier lieu doit être identifié de façon perspective. Russell aurait peut-être dit qu'il devait être un objet d'accointance. Car l'aspect persuasif du cogito dépend de la présence immédiate de son objet à l'argumentateur, personnellement présent, comme Husserl l'aurait dit. Si le cogito est une performance, cette performance particulière est comme la justice: elle ne doit pas seulement être rendue ou performée, il faut en outre voir qu'elle est rendue ou performée (dans le sens général d'en être témoin).

6. J. Hintikka, Paradigms for Language Theory and Other Essays, Dordrecht, Kluwer academic (Selected Papers; 4), 1998. Voir également J. Hintikka, Fondements d'une théorie du langage, N. Lavand (trad.), Paris, PUF (L'interrogation philosophique), 1994 (NDT). 
Ceci est étroitement lié au fait que les versions les plus persuasives du cogito utilisent la première personne du singulier «Je». Je suis un individu qui est nécessairement présent à moi-même. Affirmer «René pense, donc j'existe» n'est pas convaincant, même sorti de la bouche de René. Il faut en même temps que l'ego qui témoigne de la performance de René soit conçu comme un objet perspectif.

Dans mon deuxième article majeur sur le cogito, j'ai souligné le fait que l'entité dont Descartes pouvait espérer prouver l'existence devait être identifiée. Mais cette observation posait davantage de problèmes qu'elle n'en résolvait. Le problème le plus important concernant une reconstruction rationnelle du cogito dans ces lignes est que l'argument de Descartes ne semble pas immédiatement entraîner la conclusion qu'il veut. Car évidemment ce que Descartes veut prouver est l'existence d'une res cogitans objective, pas d'un objet perspectif éphémère. Cela ne le satisferait pas de soutenir: cette chose pense, donc cette chose existe, quelle qu'elle soit publiquement. Ce serait pourtant là toute la force de son argument si l'entité pensante et l'entité témoin n'étaient identifiées que de façon perspective.

Mais ceci semble pousser le cogito vers une erreur logique d'ambiguïté. Car dans la formule «Je pense, donc j'existe», le premier «Je» est probablement un «Je» identifié de façon perspective, tandis que le second est immédiatement poussé par Descartes vers la référence à une res (res cogitans) qui peut difficilement être autre chose qu'un objet public. Ou, peut-être de manière plus charitable, il semble pousser le cogito vers une vérité contingente. Mais que se passerait-il si le malin génie faisait oublier à Descartes son nom, le nom de celui pour qui le second «Je" est un substitut trop évident?

Ce point est d'une manière ou d'une autre obscurci par le fait que le pronom de la première personne «Je » a au moins deux usages logiquement différents. On peut l'utiliser pour faire référence soit à un objet perspectif, soit à un objet public. Le plus communément, il renvoie tout de même à un objet perspectif. Mais lorsque l'identité publique de ce «Je» devient importante, le pronom lui-même n'assume pas tout le travail. On doit de façon caractéristique avoir recours à l'usage de son nom propre. En prêtant serment, je ne dis pas seulement «je jure solennellement, ...» mais «Moi, Jaakko Juhani Hintikka, je jure solennellement». Je reviendrai sur cet aspect fâcheux de la double entente du pronom «Je». En fait, les deux sens de "Je" ne tiennent l'un à l'autre que grâce au lien constitué par le fait que j'assume que moi (le locuteur), je sais qui je suis dans le sens d'une identification publique. Le statut de cette assomption sera à nouveau considéré plus tard. 


\section{Un problème concernant les inférences entre les systèmes d'identification}

Nous voyons ici le problème de Descartes dans sa généralité. Son problème immédiat est d'utiliser l'existence de René, son moi perspectif, comme un support a priori pour asseoir l'existence d'un ego substantiel, publiquement identifié auquel nous pourrons nous référer en tant que Cartesius. Ce n'est qu'un cas particulier du problème souligné auparavant, problème qui consiste à tirer de prémisses relatives à des objets identifiés selon un mode des inférences menant à des conclusions relatives à des objets identifiés autrement. De telles inférences semblent difficiles, voire impossibles, en tant qu'inférences produites à partir des événements d'une pièce de théâtre en direction des événements de la vie réelle (et vice versa). L'argument du cogito tout entier semble erroné.

Le problème le plus profond de Descartes est de tirer une inférence à partir d'objets perspectifs vers des objets publics; à proprement parler bien sûr d'objets identifiés de façon perspective vers des objets publiquement identifiés. C'est certes un problème. Mais c'est un cas particulier d'un problème plus général. Dans les termes de mon analogie avec la pièce de théâtre, le problème se manifeste dans l'apparente impossibilité de produire des inférences basées sur ce que les acteurs font et disent lorsqu'ils jouent leur rôle dans un drame ou une comédie, pour savoir ce à quoi ils ressemblent, ou ce qu'ils voudraient être dans leur vie de tous les jours, dans le monde effectif plutôt que dans le monde imaginaire de la pièce de théâtre. Si Laurence Olivier prononce sur la scène les mots "Être ou ne pas être, telle est la question", on ne peut pas inférer que le célèbre acteur britannique envisage le suicide, seulement qu'un prince du Danemark le fait dans le monde imaginaire de Shakespeare. Et c'est ainsi que l'analogie peut sembler révéler un vice de forme décisif dans l'argument de Descartes.

Mais ce n'est pas la fin de l'histoire. Ce qu'est un personnage sur scène ou dans un film n'est pas significatif, dans tous les sens de l'expression, que pour son monde imaginaire. Il y a deux façons dont le monde réel et le monde fictionnel d'un drame peuvent, pour ainsi dire, interagir conceptuellement. Un personnage dans un film peut faire des affirmations, dans le cadre du film, qui peuvent renvoyer à de «vraies" personnes dans le sens de personnes publiquement identifiées. J'ai vu un jour un épisode d'une série télévisée dans lequel un fan déçu avait poursuivi en justice l'entraîneur de l'équipe de football des Chicago Bears, Mike Ditka, pour avoir trahi sa parole parce que l'entraîneur s'était aventuré à dire que son équipe remporterait la saison. La série avait fait l'objet d'un grand intérêt 
précisément parce que l'affirmation mise en cause dans la procédure fictionnelle renvoyait à une équipe effective, très aimée et très détestée.

\section{Se représenter soi-même}

Bien plus, parfois, une personne, ordinairement une célébrité publique, fait une apparition en jouant son propre rôle dans une pièce de théâtre ou un film. J'ai vu un jour un film sur une star de tennis imaginaire dans lequel plusieurs des plus grands joueurs du moment (John McEnroe, Pancho Gonzales, Vitas Gerulaitis, etc.) apparaissaient en tant qu'eux-mêmes. Et la série qui parlait des Chicago Bears avait un piquant spécial du fait que le coach, Mike Ditka, jouait son propre rôle dans l'épisode.

Ceci ne change pas le fait que les affirmations faites par des acteurs dans une pièce de théâtre font partie de l'action dans le monde imaginaire créé par le dramaturge ou le réalisateur du film, le cas échéant. Pourtant, elles peuvent porter sur des personnes de la vie réelle. Pour prendre un exemple supplémentaire, supposons que Clint Eastwood joue l'inspecteur Harry. Il pourrait dire, dans un tel rôle: «Dans cette situation, Clint Eastwood luimême serait incapable de garder son sérieux». L'affirmation serait portée par l'inspecteur Harry et non par Clint Eastwood, mais elle concernerait le Clint réel et non le Harry fictif. Elle devrait être vérifiée ou falsifiée en examinant le vrai Clint Eastwood, et non l'inspecteur Harry, quand bien même elle ferait partie d'un film. En conséquence, alors qu'il joue un rôle, non seulement Clint Eastwood peut affirmer quelque chose à propos de lui-même en tant que figure publique, mais il peut aussi rendre fausse cette affirmation en faisant ou en affirmant quelque chose. Dans mon exemple, il pourrait prouver que cette affirmation est fausse en maintenant sa lèvre supérieure immobile. Dans l'autre exemple, John McEnroe pourrait dire: "John McEnroe lui-même ne pourrait par renvoyer cette balle» et invalider cette formule en renvoyant la balle. D'où il est clair et net que ce qui se produit dans un film ou une pièce de théâtre, en tant que partie de l'action du film ou de la pièce, peut démontrer quelque chose à propos du monde réel.

\section{Pourquoi le cogito est-il a priori?}

Mais ceci génère-t-il vraiment un argument $a$ priori? Plus haut, j'ai souligné que ce «Je» s'appuyait d'abord sur un objet identifié de façon perspective. Cet objet est de facto identique à un objet publiquement identifié, c'est-àdire celui qui porte son nom. Alors, pourquoi notre philosophe ne peut-il soutenir: «Moi, René Descartes, je pense, donc moi, René Descartes, 
j'existe»? Nous en voyons la raison: l'identité de René (le «Je» identifié de façon perspective) et Cartesius (la personne publique) semble n'être que contingente.

Ce qui se passe, c'est que les deux systèmes d'identification sont corrélés au même système de référence. Et c'est une autre propriété de la logique $\mathrm{du}$ «Je » que de pouvoir être utilisé pour faire référence à un objet identifié publiquement, c'est-à-dire qu'un objet public est identique avec l'objet perspectif «Je». D'où l'idée selon laquelle l'argument pourrait ainsi se déployer de la façon suivante: «Moi (l'objet identifié de façon perspective) j'existe, donc qui que je sois (publiquement identifié), j'existe aussi». Bien plus, le fait que cet objet public soit nommé Cartesius peut alors servir en tant que vérité conceptuelle supplémentaire qui autorise René à penser que sa conclusion se rapporte à une res actuelle (c'est-à-dire publiquement identifiée). Et je suis cette res particulière pour la même raison qu'un certain acteur est (joue le rôle de) tel ou tel personnage. Le fait crucial est que la relation de nomination est évidemment une affaire conceptuelle (linguistique), et non une affaire contingente (factuelle).

Ce point mérite d'être souligné. Force est de constater qu'une inférence du type du cogito dépend de la connaissance de qui représente qui. Je dois savoir que l'inspecteur Harry est joué par Clint Eastwood ou que John McEnroe est joué par lui-même pour comprendre l'influence performative de ce qu'ils font. Mais, je le souligne : cette identification est une vérité conceptuelle. Le besoin de cette information concerne donc le personnage a priori (on pourrait dire personnage conceptuel ou même nécessaire) de l'argument. Ce n'est pas une vérité contingente. Que tel acteur tienne tel rôle fait partie de la définition de la pièce ou plutôt de la production particulière de la pièce. Pour établir l'identité d'un acteur et d'un personnage dans une pièce vous n'avez pas à entreprendre de recherche dans les archives de l'histoire théâtrale. Il suffit de lire l'affiche. Une démonstration théâtrale de quelque chose qui concerne le monde réel est ainsi dans un sens parfaitement rigoureux une vérité conceptuelle.

Mais est-ce que cette identité analogue tient aussi réellement, entre les rôles d'un objet perspectif et d'un objet public, à des raisons purement conceptuelles? Est-ce que ces rôles sont reliés conceptuellement de la même manière qu'un acteur ou une actrice le sont au personnage qu'il ou elle joue? Dans la plupart des cas, ils ne le sont pas. Une affirmation exacte comme: "Cet homme est le maire Menino » n'est pas seulement vraie pour des raisons conceptuelles. Mais il y a un cas dans lequel cette identité se tient. C'est celui du pronom de la première personne du singulier «Je». Comme je l'ai indiqué tout à l'heure, dans l'un de ses usages typiques, une identification perspective et une identification publique coïncident 
per definitionem, en dépit de la tension qu'il y a entre la signification de l'identification perspective et celle de l'identification publique. Si je me présente à une nouvelle connaissance en disant: «Je suis Jaakko Hintikka», je n'affirme pas un fait à propos du monde, mais une vérité conceptuelle, en un sens linguistique. Je vous révèle mon nom. D'où l'idée selon laquelle les rôles des identifications perspective et publique sont conceptuellement connectés dans le cas de «Je» ou «Moi». C'est sémantiquement l'une des propriétés les plus caractéristiques de ce pronom, une propriété parfaitement ajustée pour le propos de Descartes.

Mais une objection va être soulevée ici. Il a été indiqué que, même dans le cas du pronom de la première personne "Je", les deux modes d'identification n'allaient ensemble qu'en vertu du fait que la personne qui se représente elle-même sait qui il ou elle est selon les critères publics. Un amnésique peut ne pas y parvenir. Toutefois, cela n'empêche nullement que la connexion soit une connexion conceptuelle. Ce qu'un amnésique oublie n'est pas un fait empirique mais une vérité conceptuelle, c'est-àdire la signification de son nom. Je peux oublier mon nom, mais aussi longtemps que je parle français, je sais que je peux faire référence à mon moi publiquement identifié (et pas seulement à mon incarnation identifiée de façon perspective) en disant "Je».

Ceci montre par incidence ce qui est vrai et faux dans la notion de désignation rigide de Kripke. Il a perçu le sens dans lequel un nom propre faisait référence à ce à quoi il fait référence pour des raisons conceptuelles. Mais ce à qui il fait référence dans un autre monde (scénario) peut seulement être déterminé sur la base de ce scénario, et ne peut pas être déterminé en lui assignant une référence dans le monde actuel. C'est exactement ce qui se passe avec l'identité du «Je» de René et de Cartesius. Elle tient durant la production de la "pièce du cogito». Elle ne dit rien de celui qui, dans une autre production, devrait énoncer «Je» pour faire référence à Cartesius.

\section{Le «donc» du cogito}

Ces aperçus des différentes distinctions entre des modes séparés mais coexistants d'identification nous autorisent finalement à résumer la logique du cogito. Descartes produit pour ainsi dire une saynète dans laquelle il joue lui-même. Supposons que le personnage qu'il joue soit nommé René. René peut alors dire, dans le cadre de la pièce, "Cartesius existe». En disant cela, il démontre l'existence du personnage nommé René. Ceci est analogue au fait qu'il rende son existence évidente à travers l'acte de pensée qui consiste à considérer sa propre existence en tant qu'objet perspectif (objet de sa pensée). Ceci n'implique pas encore l'existence de Cartesius, 
le philosophe publiquement identifié. Mais René est, en dehors de la pièce, la même personne que Cartesius. Donc l'acte de pensée de René démontre l'existence de Cartesius, au moyen de l'assomption additionnelle que l'acteur qui joue René est bel et bien Cartesius. Et ce n'est pas une vérité contingente à propos des identités de facto, mais une vérité déterminée par l'attribution des rôles qui fait partie de la définition de la pièce en question ou, pour parler strictement, de cette production particulière de la pièce.

De façon alternative, mais équivalente, le rôle de René que Descartes a joué dans son drame mental était celui d'un sceptique qui essaie de nier toute chose. Quand il a étendu son doute à son existence et qu'il l'a exprimé en affirmant à lui-même "Je n'existe pas», il a alors démontré non seulement l'existence d'un personnage de pièce mais aussi de la personne publique qui jouait dans ce rôle. Le cogito peut être compris de l'une ou l'autre de ces manières.

\section{Mais est-ce l'argument de Descartes?}

Cette interprétation du cogito cartésien pourra peut-être sembler trop tirée par les cheveux. Mais est-elle réellement artificielle? Il n'est pas exagéré d'imaginer que Descartes conçoive effectivement son argument en termes de jeu de rôle:

De même que les comédiens, attentifs à ne pas laisser voir la rougeur sur leur front, mettent un masque; de même moi, au moment de monter sur le théâtre du monde, où je me suis tenu jusqu'ici en spectateur, je parais masqué 7 .

L'argument du cogito faisait-il partie du rôle sur la scène de la vie que Descartes assumait et celui-ci a-t-il porté le masque de René le pyrrhonien par intermittence?

\section{Le «scepticisme» de Descartes}

Par ailleurs, l'interprétation présentée ici aide à mettre en perspective la méthode du doute de Descartes et sa relation au scepticisme. En quel sens Descartes doutait-il ? Pas dans le sens de ses vues philosophiques établies qui devaient être l'aboutissement de ses arguments, y compris le cogito. Au contraire, il voulait prouver son essence en tant que substance

7. «Ut comcedi, moniti ne in fronte appareat pudor, personam induunt: sic ego, hoc mundi theatrum conscensurus, in quo hactenus spectator exstiti, larvatus prodeo", Cogitationes privatce, AT X, 213; R. Descartes, Étude du bon sens. La recherche de la vérité et autres écrits de jeunesse, V. Carraud, G. Olivo (éd. et trad.), Paris, PUF (Épiméthée), 2013, p. 65. 
pensante, l'existence de Dieu, et la véracité des idées claires et distinctes, entre autres choses.

$\mathrm{Au}$ lieu de professer le scepticisme ou le «pyrrhonisme», comme cela a pu être nommé, Descartes utilise une ligne de pensée sceptique contre le scepticisme, mais la pousse jusqu'à sa propre limite. Cette sorte d'argument présuppose inévitablement que l'argumentateur parle pour ainsi dire à deux voix. Si René avait dû mener une enquête normale déterminée et avait soudainement affirmé quelque chose de contraire à ce qu'il se serait affirmé à lui-même auparavant, nous devrions dire qu'il a seulement changé d'avis. Ce qu'il aurait dit avant cette volte-face, quoi que ce soit, n'aurait pas automatiquement été pertinent pour ses nouvelles vues.

Mais bien sûr ce n'est pas ainsi que procède Descartes. Il a besoin d'un alter ego, appelons-le Cartesius, pour témoigner de l'expérience de pensée sceptique de René et en tirer une conclusion. D'où il s'ensuit que parler (ou plutôt penser) à deux voix différentes est l'un des aspects constants de la méthode du doute de Descartes. Il n'avait pas à être un sceptique sérieux, si ce n'est dans le rôle qu'il jouait.

En d'autres termes, Descartes n'a pas adopté lui-même littéralement les vues ou arguments sceptiques; il a laissé René les assumer.

Je me resolu de feindre que toutes les choses qui m'estoient jamais entrées en

l'esprit, n'estoient non plus vrayes que les illusions de mes songes ${ }^{8}$.

Une telle affirmation équivaut à passer du monde cognitif ordinaire de Cartesius à la pièce mentale dans laquelle joue René le pyrrhonien radical. Quoi qu'il en soit, ce serait une erreur de dire que René, en jouant ce rôle, ne faisait que considérer la possibilité du scepticisme. René devait soutenir ces arguments dans le but de rendre leur fausseté évidente.

Un commentateur érudit et perspicace va jusqu'à dire à propos de l'Aufhebung du scepticisme de Descartes: "Ce stupéfiant changement de situation n'était possible que parce que le pyrrhonisme avait été pris suffisamment au sérieux [par Descartes]» 9 .

Ceci peut paraître paradoxal, en ce que sa position sceptique n'est en un sens pas prise sérieusement comme étant sa propre position, mais plutôt assumée en tant que point de départ d'une expérience de pensée. L'analogie de la pièce de théâtre montre comment les deux aspects de l'apparent

8. Discours de la méthode, IV, AT VI, 32.

9. R. H. Popkin, The History of Scepticism: From Erasmus to Descartes, Assen, Van Gorcum, 1960, p. 193; éd. revue et augmentée: The History of Scepticism: From Savonarola to Bayle, Oxford, Oxford University Press, 2003, p. 155. 
paradoxe peuvent être tenus ensemble. C'est René qui devait prendre le pyrrhonisme au sérieux, pas Cartesius qui était le critique du scepticisme.

C'est probablement cette dualité des rôles qui fait que les contemporains de Descartes l'ont facilement pris pour un sceptique. Ils n'ont pour ainsi dire pas réalisé que ce que René disait ne traduisait pas les vues établies de Descartes.

\section{La pièce du dénombrement de Gödel}

Une autre objection importante est que la logique que j'ai attribuée à Descartes peut sembler ad hoc. La réponse à cette objection est qu'il n'y a rien d'ad hoc au problème général qui lui a été attribué. Le problème concerne la possibilité de raisonner à partir d'objets identifiés d'une manière en vue des mêmes objets identifiés d'une autre manière. Pour aider à dissiper l'impression de caractère $a d$ hoc, nous pouvons présenter un autre cas de l'usage du jeu de rôle dans le but de produire des inférences relatives à des objets identifiés de deux manières différentes. Dans cet autre cas d'étude, les «acteurs» ne sont pas humains, mais sont des nombres naturels, et le nom de la "pièce» dans laquelle on leur attribue des rôles peu familiers est le dénombrement de Gödel ${ }^{10}$. Cette «pièce» est utilisée par Gödel pour prouver son premier fameux théorème de l'incomplétude.

On a souvent dit - Gödel lui-même l'a parfois dit - que le raisonnement de Gödel pour prouver ce théorème était comparable au raisonnement compris dans le paradoxe du menteur ${ }^{11}$. Ceci est une grossière erreur. Les nombres ne peuvent pas se référer à eux-mêmes. Il n'y a pas de pronom de la première personne dans le langage de l'arithmétique et aucun autre moyen d'autoréférence directe. À un moment donné, Wittgenstein a essayé d'envisager une extension de notre notation arithmétique dans laquelle une telle autoréflexivité était possible. Ceci aurait sans aucun doute rendu inconsistant le langage mathématique en question.

La sorte d'autoréférentialité qui se trouve dans l'argument de Gödel est de la même sorte qu'une référence théâtrale dans une pièce jouée pour soimême en tant que personne ordinaire à l'extérieur du théâtre. Dans la pièce de Gödel, des nombres ordinaires honnêtes se voient assigner des rôles de codification de déclarations numériques ou d'autres formules, dans le même sens qu'une personne particulière peut être en même temps Sir Laurence

10. Voir J. Hintikka, On Gödel, Belmont, Wadsworth - Thomson Learning (Wadsworth philosophers series), 2000.

11. K. Gödel, «Über formal unentscheidbare Sätze der Principia Mathematica und verwandter Systeme I», Monatshefte für Mathematik und Physik, 38, 1931, p. 173-198; trad. fr. par J.-B. Scherrer dans K. Gödel, Le théorème de Gödel, E. Nagel, J. R. Newmann, K. Gödel (éd.), Paris, Seuil (Sources du savoir), 1989, p. 105-143. 
Olivier et le prince Hamlet. Ce n'est que dans ce rôle qu'ils peuvent faire référence à des nombres, y compris à eux-mêmes. On a prétendu (par exemple Burton Dreben) que le fameux théorème de Gödel n'était qu'un théorème élémentaire concernant les nombres naturels. C'est vrai, mais cela ne veut pas dire que dans son rôle dramatique il peut également être un résultat métalogique à propos des limites de la prouvabilité formelle des vérités arithmétiques.

Gödel considère un certain prédicat arithmétique formellement défini $\mathrm{F}(\mathrm{x})$. Par un ingénieux raisonnement, il construit un nombre $g$ qui va jouer dans sa pièce le rôle de $\mathrm{F}(\mathrm{g})$. Astucieusement, il a sélectionné $\mathrm{F}(\mathrm{x})$ de telle manière qu'en jouant son rôle dans la pièce il dit (traduit en français): «La proposition contenant le nombre $x$ de Gödel n'est pas possible». Bien sûr, $\mathrm{F}(\mathrm{x})$ est, durant sa journée de travail, un prédicat arithmétique sans problème. Mais (en assumant la consistance de l'arithmétique) $g$ ne peut alors pas codifier une fausse déclaration, car sa négation dit qu'elle est prouvable (consistance signifie que les déclarations prouvables sont vraies). D'où il s'ensuit que c'est vrai mais improuvable, car c'est ce qui est dit en vérité. Tel est le résultat de Gödel.

Ainsi, l'apparente autoréférence dans l'argument de Gödel est en réalité une inférence opérée à partir d'objets identifiés d'une manière en vue des mêmes objets envisagés selon un autre mode, tout comme l'inférence de l'existence de René est tirée de celle de son alter ego Cartesius.

Ceci illustre également le fait que l'argument de Descartes ne dépend pas en dernière analyse des particularités de la logique des pronoms de la première personne. (Comme je l'ai indiqué, il n'y a pas de pronoms de la première personne en arithmétique.) Descartes fait ses affaires en adoptant le comportement logique du "Je», mais il n'en a pas besoin pour son argument. Le cogito n'est pas qu'un théorème relevant de la logique du «Je».

L'analogie du jeu de rôle fournit également une représentation plus précise du raisonnement de Gödel que la plupart des discussions philosophiques portant sur sa signification. En même temps, elle montre la similitude essentielle que la ligne de pensée de Gödel entretient avec celle de Descartes.

Jaakko HinTıKKA

Boston University

Traduction de Xavier KIEFT 